\title{
ADVERSE DERMATOLOGIC AND RESPIRATORY HEALTH PROBLEMS AMONG STREET SWEEPER'S WORKERS: A COMPARATIVE STUDY
}

\author{
By \\ Hassan $\mathrm{O}^{1}$, Abed $\mathrm{H}^{1}$, Araby $\mathrm{E}^{1}$ and Fayed $\mathrm{N}^{2}$ \\ ${ }^{1}$ Department of Community Medicine, ${ }^{2}$ Department of Dermatology and Andrology, Faculty of Medicine, \\ Benha University, Benha, Egypt.
}

Hassan O: omayma.hassan@fmed.bu.edu.eg

ArabyE:eman.attia@fmed.bu.edu.eg
Abed H: hala.hassan@fmed.bu.edu.eg

Fayed H: nahlafayed@yahoo.com

\begin{abstract}
Introduction: Street Sweeping plays an important role in maintaining the health and hygiene in cities. This job exposes street sweepers to a variety of risk factors that leads to multiple health hazards such as: respiratory system problems, cardiovascular degeneration, musculoskeletal disorders, infections, skin and gastrointestinal problems. Aim of work: To study the prevalence of some dermatological and respiratory manifestations among street sweepers and its relevance with occupational exposure. Materials and methods: The present study is a comparative cross-sectional study, conducted at Benha city, Qualubya Governorate, Egypt. Data was collected over a period of 4 months. Cluster random sampling was used to choose the study groups as we choose 5 quarters out from 16 quarters in Benha city by simple random sample. The total number of participant enrolled in this study was 200 individuals (126 street sweepers as a study group and 74 administrative staff as a control), all were nonsmokers, matched for duration of work and age. Results: Significant higher percentage of skin disorders was detected among street sweepers in comparison to administrative staff: hair loss (19\% vs. 6.8\%), scabies (15.1\% vs. $4 \%$ ), frunculosis (18.3\% vs. $4.1 \%)$, contact dermatitis (17.5\% vs. $2.7 \%$ ), infected wound (11\% vs. $1.4 \%$ ), and varicosities $(25.4 \%$ vs. $6.8 \%)(\mathrm{p}<0.01)$. Pulmonary function tests of street sweepers showed significant decline of (FEV1, FVC and FEV1/FVC ratio) in comparison to the control group. Our study revealed strong association between working for 10 years or more and development of chronic cough, phlegm ( $\mathrm{p}$ value $<0.01$ ), wheezes and feeling of shortness of breath ( $\mathrm{p}$ value $<0.05$ ). More than fifty percent of street sweepers were not
\end{abstract}


wearing personal protective clothes at all. Conclusion: Exposure of sweeper's workers to many health hazards and poor use of protective equipments are associated with skin and respiratory diseases.

Keywords: Street sweeper's workers, Skin diseases, Respiratory diseases, Protective equipments and Benha city.

\section{Introduction}

In recent decades, urbanization became a widespread problem in developing countries with rapid economic development. It causes in accumulation of population in cities that resulted in huge number of sanitation problems as a lot of garbage, dirty streets and blocked drains which greatly expand the sanitation specialist workload and working hours (Chandler and Feuille, 1994).

Street Sweeping plays an important role in maintaining the health and hygiene in cities. It is a vigorous task that involves sweeping of roads, parks, markets and open areas. This occupation exposes street sweepers to a variety of risk factors as dust, ultraviolet rays, toxins, sharps, microorganisms, volatile organic matter, mechanical stress, bioaerosols and diesel exhaust pollution (Krajewski et al., 2002).

At present, the management standards and norms of municipal solid wastes in industrialized countries have significantly diminished occupational health impacts. However, in developing countries, solid waste management still needs to be addressed. There is little, if any, workers protection from direct contact, injury and virtually no dust control at the workplace (CointreauLevine, 1995).

Apart from the social marginalization that these workers face, there is insufficient health care, and this makes them more prone to specific health problems due to their occupation which include respiratory system problems, cardiovascular diseases, musculoskeletal disorders, infections, skin problems and gastrointestinal problems (Tamara et al., 2008).

Intact skin serves as a natural barrier between the internal and external environment, protecting the body from pathogens and damage (Nayak et al., 2013).

As regards the respiratory system, many studies have postulated association between increased respiratory illness, abnormal lung function tests and working at open dumps (CointreauLevine et al.,1998; Athanasiou et 
al.,2010).According to Center of Disease Control (CDC), chronic obstructive pulmonary disease (COPD) causes $3.09 \%$ of total Disability Adjusted Life Years(DALYs)worldwide. In Egypt, it is the fourth cause of death among the Egyptian population. (GBD, 2010).

Exposure to organic dusts containing high concentrations of biologically active agents, bacteria and fungi, gases and bio-aerosols; increases the risk for development of respiratory disease (CointreauLevine, 2006).Also, waste collection is conducted in high traffic density, and in developing countries, vehicle emissions are not controlled. Pollutants which affect the respiratory system are generally beyond the body's natural cleaning mechanisms such as muco-ciliary clearance and are likely to be retained (Seaton, 1995).

Various socioeconomic factors such as poverty, lack of education, poor housing conditions and poor diet affect medical problems of those workers (Nagaraj et al., 2004).

Few researches were done in Egypt to study the health profile of these workers. Previous studies had predominantly focused on musculoskeletal disorders, sharp injuries and risk of hepatitis (Abou-ElWafa et al.,2012; Ewis et al.,2013; Abd El-Wahab et al., 2014 ;).

\section{Aim of work}

To study the prevalence of some dermatological and respiratory manifestations among street sweepers and its relevance with occupational exposure.

\section{Materials and methods}

Study design: It is a comparative cross-sectional study

\section{Place and duration of the study:} The study is conducted at Benha city, Qualubya Governorate, Egypt. Data was collected over a period of 4 months (from the beginning of March till the end of June 2017).

Study sample: Cluster random sampling was used to choose the study groups as we choose 5 quarters (Atrrep ,El Mansheia, Benha ElGededa, Ezbet El Zeraa and Kafer El Gazar) out from 16 quarters in Benha city by simple random sample, then all workers working at these quarters were asked to participate in this study .

- Exposed group: one hundred and twenty-six street sweepers out from 263 sweepers agreed to participate in the study.Inclusion criteria: they are working as street sweepers for at least 10 years and they are non smokers. 
- Non- Exposed group: Total of 74 of administrative officers from Benha University Hospital. Inclusion criteria: They are not engaged in street sweeping, nonsmokers, had no past medical or family histories of respiratory illnesses, chest operations or injuries and matched the exposed group for duration of work and age.

\section{Study methods:}

1-An interview structured questionnaire based on data from relevant literature was used to collect data from street sweepers.Data were collected using data plan schedule; all interviews were conducted face to face by the researchers.

Socio-demographic information was obtained through questionnaire about marital status; level of education and duration of employment were collected from both groups. Full detailed occupational history, weight and height also were recorded; Body Mass Index(BMI)was calculated.

Uses of personnel protective equipments (PPE): the closed-ended questions to assess availability of PPEs and level of wearing them during working among exposed group. The respondents were asked to answer "Yes" or "NO" on list of PPEs, gloves, eye protection, foot protection and mask by the investigator.

2-Modified American Thoracic Society Respiratory Symptoms Questionnaire was used to collect data about respiratory health symptoms (Comstock et al., 1979).

The questionnaire arranged to ask about cough, phlegm, wheezes, breathlessness, and duration of exposure, past respiratory illness and past dust exposure.

3- Ventilatory functions: Forced Vital Capacity (FVC), Forced Expiratory Volume 1 (FEV1) and FEV1/FVC were measured using spirometer, JAEGER master screen pulmonary function test (MS-PFT analyzer unit). The best trial measurement was recorded after 3 successive trials. Subjects who did not perform an acceptable maneuver after three attempts were excluded from the study. Predicted values were calculated according to subject's gender, age and height. Spiro metric values (FVC, FEV1and FEV1/FVC) less than $80 \%$ of predicted values were considered reduced. Data collection and lung functions measurements were carried out in Chest department of Benha University Hospital. 
4- Skin examination was done to detect dermatosis especially on face, neck, arms, hands, and feet which are the most exposed skin areas in their occupational environment, skin manifestations that including: hair loss, scabies, frunculosis, contact dermatitis, vitiligo, infected wound, foot fissures, and skin allergy, varicosity and pigmentation spots. The presence or absence of the disease, the severity of illness and the personal history of skin diseases were recorded, especially specific occupational skin diseases. Professional dermatologists designed the interview questionnaire.

\section{Pre-testing:}

Respiratory health symptoms questionnaire and utilization of PPEs data collection tools were tested by selection of six street sweepers, their responses were not included in the analysis, they were from Azbat El Noor quarter in Benha areas. It was done to find how the study population understood the questionnaire and to improve reliability in data collection.

\section{Consent}

An informed written consent (in Arabic language) was obtained from the participants. It included their per- sonal data and details about the study as title, objectives, methodology, expected benefits and risks, and confidentiality of data.

\section{Ethical approval}

An approval from The Research Ethics Committee in Benha Faculty of Medicine was obtained to carry out this work. Also, verbal approval from city council was obtained to guarantee cooperation of workers and to facilitate our work.

\section{Data management}

The collected data were tabulated and analyzed using SPSS version 20 software. Qualitative data were expressed as frequencies and percentages, while continuous variables were presented as Mean \pm Standard Deviation and Range. Chi square "X2" was used to detect the association between respiratory and skin manifestations and exposure to hazards of street sweeping. Continuous data were tested for normality using Kolomogrov-Smirnov test as proved to be parametric, continuous variables were analyzed independent t test and Pearson's correlation coefficient (rho). Two-sided $\mathrm{p}$ value $\leq 0.05$ was considered significant. 


\section{Results}

Table (1): Socio-demographic and anthropometric data of the studied groups.

\begin{tabular}{|c|c|c|c|}
\hline Characteristics & $\begin{array}{l}\text { Sweepers } \\
(\mathrm{No}=126)\end{array}$ & $\begin{array}{l}\text { Control } \\
(\mathrm{No}=74)\end{array}$ & $\begin{array}{c}\text { Tests of } \\
\text { significance \& } \\
\text { p value }\end{array}$ \\
\hline $\begin{array}{l}\text { Age(Years) } \\
\text { Range } \\
\text { Mean } \pm \text { SD }\end{array}$ & $\begin{array}{c}25-56 \\
40.9 \pm 8.6 \\
\end{array}$ & $\begin{array}{c}29-56 \\
42.6 \pm 7.0 \\
\end{array}$ & $\begin{array}{l}\mathbf{t}=1.5 \\
(0.14)\end{array}$ \\
\hline $\begin{array}{l}\text { Marital status } \\
\text { Single } \\
\text { Married } \\
\end{array}$ & $\begin{array}{l}31(24.6) \\
95(75.4)\end{array}$ & $\begin{array}{l}10(13.5) \\
64(86.5) \\
\end{array}$ & $\begin{array}{c}\mathbf{X}^{2}=3.5 \\
(0.06)\end{array}$ \\
\hline $\begin{array}{l}\text { Residence } \\
\text { Urban } \\
\text { Rural } \\
\end{array}$ & $\begin{array}{l}64(50.8) \\
62(49.2)\end{array}$ & $\begin{array}{c}70(94.6) \\
4(5.4) \\
\end{array}$ & $\begin{array}{c}X^{2}=40.5 \\
(\mathbf{0 . 0 0 )} * *\end{array}$ \\
\hline $\begin{array}{l}\text { Education } \\
\text { Illiterate } \\
\text { Illiterate certificate } \\
1^{\text {ry }} \text { education } \\
\text { Prep. education } \\
\text { Higher education }\end{array}$ & $\begin{array}{c}76(60.3) \\
18(14.3) \\
25(19.8) \\
7(5.6) \\
0(0) \\
\end{array}$ & $\begin{array}{c}0(0) \\
0(0) \\
0(0) \\
0(0) \\
74(100)\end{array}$ & $\begin{array}{c}\mathbf{F E T}=245 \\
(\mathbf{0 . 0 0 )} * *\end{array}$ \\
\hline $\begin{array}{l}\text { BMI }\left(\mathbf{K g} / \mathbf{m}^{2}\right) \\
\text { Range } \\
\text { Mean } \pm \text { SD }\end{array}$ & $\begin{array}{l}18-33.3 \\
24.6 \pm 3.2\end{array}$ & $\begin{array}{c}17.6-46 \\
30.2 \pm 4.7\end{array}$ & $\begin{array}{c}\mathbf{t}=9.9 \\
(\mathbf{0 . 0 0 )} * *\end{array}$ \\
\hline $\begin{array}{l}\text { Underweight } \\
\text { Normal weight } \\
\text { Overweight } \\
\text { Obese }\end{array}$ & $\begin{array}{c}6(4.8 \%) \\
48(38.1 \%) \\
67(53.2 \%) \\
5(3.9 \%) \\
\end{array}$ & $\begin{array}{c}2(2.7 \%) \\
5(6.8 \%) \\
25(33.8 \%) \\
42(56.7 \%)\end{array}$ & $\begin{array}{l}\mathbf{F E T}=80 \\
(\mathbf{0 . 0 0}) * *\end{array}$ \\
\hline $\begin{array}{l}\text { Duration of work: } \\
\text { Range (Years) } \\
\text { Mean } \pm \text { SD }\end{array}$ & $\begin{array}{c}5-30 \\
14.7 \pm 7.0\end{array}$ & $\begin{array}{c}5-30 \\
14.9 \pm 7.0\end{array}$ & $\begin{array}{c}\mathbf{t}=0.19 \\
(0.8)\end{array}$ \\
\hline
\end{tabular}

**Highly statistically significant

FET: Fisher Exact Test

BMI: Body Mass Index 


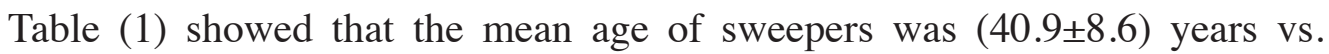
$(42.6 \pm 7.0)$ of the control group. About $75 \%$ of sweepers were married vs. $86.5 \%$ of control group. As regards residency, percentage of urban residents were more than rural ones in both groups but still it is higher among administrative staff (94.6\% vs. 50.8\%).All administrative staff was highly educated while sixty percent of sweepers was illiterate. The majority of sweepers was normal or overweight ( $38.1 \% \& 53.2 \%$ respectively) while the majority of the control group was overweight or obese $(33.8 \% \& 56.7 \%$ respectively). The mean of work duration for the sweepers is nearly the same, $(14.7 \pm 7.0)$ vs. $(14.9 \pm 7.0)$ for the control group. There were statistically significant differences between both groups regarding residence, educational level and BMI $(\mathrm{P}<0.01)$. 
Table (2): Association between skin manifestations, socio-demographic and occupational factors of street sweepers.

\begin{tabular}{|c|c|c|c|c|c|c|}
\hline & $\begin{array}{c}\text { Age } \\
(\geq \mathbf{4 0} /<40 \mathrm{yrs}\end{array}$ & $\begin{array}{c}\text { Marital } \\
\text { status (single/ } \\
\text { married) }\end{array}$ & $\begin{array}{c}\text { Residence } \\
\text { (rural/ urban) }\end{array}$ & $\begin{array}{c}\text { Educational } \\
\text { level } \\
\text { (not educated/ } \\
\text { educated) }\end{array}$ & $\begin{array}{c}\text { Duration of } \\
\text { work } \\
(\geq 10 \mathrm{yrs} /<10 \\
\text { yrs })\end{array}$ & $\begin{array}{c}\text { Wearing PPE } \\
\text { (not wearing } \\
\text { /wearing) }\end{array}$ \\
\hline $\begin{array}{l}\text { Hair loss } \\
\mathbf{X}^{2} \text { (pvalue) } \\
\text { OR:CI }\end{array}$ & $\begin{array}{l}0.15(>0.05) \\
0.84: 0.3-2.1\end{array}$ & $\begin{array}{c}0.23(>0.05) \\
0.77: 0.26-2.3\end{array}$ & $\begin{array}{c}0.14(>0.05) \\
0.85: 0.35-2.1\end{array}$ & $\begin{array}{l}0.06(>0.05) \\
1.1: 0.45-2.8\end{array}$ & $\begin{array}{c}\text { FET } \\
0.2(>0.05) \\
1.31: 0.4-4.2\end{array}$ & $\begin{array}{c}0.05(>0.05) \\
0.9: 0.4-2.2\end{array}$ \\
\hline $\begin{array}{l}\text { Scabies } \\
X^{2} \text { (p value) } \\
\text { OR-CI }\end{array}$ & $\begin{array}{c}0.04(>0.05) \\
0.9: 0.3-2.4\end{array}$ & $\begin{array}{c}\text { FET }(0.04) \\
\quad(>0.05) \\
1.1: 0.4-3.4\end{array}$ & $\begin{array}{l}\mathbf{1 4 . 5}(<\mathbf{0 . 0 1})^{*} \\
11.7: 2.6-53.3\end{array}$ & $\begin{array}{l}\mathbf{7 . 9}(<\mathbf{0 . 0 1})^{*} \\
6.9: 1.5-31.4\end{array}$ & \begin{tabular}{|c|} 
FET \\
$\mathbf{0 . 0 2}(<\mathbf{0 . 0 5})^{*}$ \\
$0.09: 0.28-3.04$
\end{tabular} & $\begin{array}{c}0.08(>0.05) \\
1.2: 0.4-3.2\end{array}$ \\
\hline $\begin{array}{l}\text { Frunculosis } \\
\mathbf{X}^{2} \text { (p value) } \\
\text { OR-CI }\end{array}$ & $\begin{array}{l}0.04(>0.05) \\
1.2: 0.44-2.7\end{array}$ & $\begin{array}{l}0.12(>0.05) \\
0.82: 0.3-2.4\end{array}$ & $\begin{array}{c}\text { 12.6( }<\mathbf{0 . 0 1}) * \\
6.6: 2.1-20.9\end{array}$ & $\begin{array}{l}\text { 11.3(<0.01)* } \\
9.2: 2.04-41.1\end{array}$ & $\begin{array}{c}\text { FET } \\
2.19(>0.05) \\
3.0: 0.66-13.8\end{array}$ & $\begin{array}{c}0.28(>0.05) \\
1.3: 0.5-3.3\end{array}$ \\
\hline $\begin{array}{l}\text { Contact } \\
\text { dermatitis } \\
\left.X^{2} \text { (p-value }\right) \\
\text { OR-CI }\end{array}$ & $\begin{array}{l}0.001(>0.05) \\
0.99: 0.39-2.5\end{array}$ & $\begin{array}{c}0.05(\mathrm{p}>0.05) \\
0.88: 0.3-2.6\end{array}$ & $\begin{array}{c}\mathbf{1 1 . 3}(\mathbf{p}<\mathbf{0 . 0 1}) * \\
6.1: 1.5-18.7\end{array}$ & $\begin{array}{l}\mathbf{1 0 . 4}(<\mathbf{0 . 0 1})^{*} \\
8.6: 1.9-38.6\end{array}$ & $\begin{array}{c}\text { FET } \\
1.9(>0.05) \\
2.8: 0.62-13.1\end{array}$ & $\begin{array}{l}\mathbf{7 . 6}(<\mathbf{0 . 0 1})^{*} \\
5.2: 1.5-18.7\end{array}$ \\
\hline $\begin{array}{l}\text { Vitiligo } \\
\text { X}^{2} \text { (p-value) } \\
\text { OR-CI }\end{array}$ & $\begin{array}{c}0.26(>0.05) \\
1.4: 0.4-4.4\end{array}$ & $\begin{array}{c}\text { FET } \\
0.66(\mathrm{p}>0.05) \\
0.53: 0.11-2.5\end{array}$ & $\begin{array}{l}2.3(p>0.05) \\
2.5: 0.74-8.8\end{array}$ & $\begin{array}{l}3.6(\mathrm{p}>0.05) \\
4.1: 0.9-19.2\end{array}$ & $\begin{array}{c}\text { FET } \\
0.18(\mathrm{p}>0.05) \\
1.4: 0.3-6.8\end{array}$ & $\begin{array}{c}\mathbf{6 . 2}(\mathbf{p}<\mathbf{0 . 0 1}) * \\
9.2: 1.2-73\end{array}$ \\
\hline $\begin{array}{l}\text { Infected } \\
\text { wound } \\
\text { X }^{2} \text { (p-value) } \\
\text { OR-CI }\end{array}$ & $\begin{array}{c}0.03(>0.05) \\
1.1: 0.3-3.4\end{array}$ & $\begin{array}{c}\text { FET } \\
0.13(\mathrm{p}>0.05) \\
1.3: 0.36-4.3\end{array}$ & $\begin{array}{l}\text { 12(p<0.01)* } \\
16.7: 2.1-132\end{array}$ & $\begin{array}{c}\mathbf{6 . 9}(\mathbf{p}<\mathbf{0 . 0 1}) * \\
10: 1.3-80\end{array}$ & $\begin{array}{c}\text { FET } \\
0.31(\mathrm{p}>0.05) \\
1.6: 0.3-7.4\end{array}$ & $\begin{array}{c}\mathbf{4 . 7}(\mathbf{p}<\mathbf{0 . 0 1})^{*} \\
4.8: 1.0-22.6\end{array}$ \\
\hline $\begin{array}{l}\text { Foot fissures } \\
X^{2}(p-v a l u e) \\
\text { OR-CI }\end{array}$ & $\begin{array}{c}\text { FET } \\
0.08(>0.05) \\
0.8: 0.19-3.4\end{array}$ & $\begin{array}{c}\text { FET } \\
0.001(>0.05) \\
1.0\end{array}$ & $\begin{array}{c}\text { FET } \\
(\mathbf{8 . 8 )}<\mathbf{0 . 0 1} * \\
8.3\end{array}$ & $\begin{array}{c}\text { FET } \\
2.64(\mathrm{p}>0.05) \\
5.0: 0.6-41.7\end{array}$ & $\begin{array}{c}\text { FET } \\
0.29(\mathrm{p}>0.05) \\
1.8: 0.2-15.2\end{array}$ & $\begin{array}{c}\text { FET } \\
5.62(\mathrm{p}<0.05) \\
5.0: 0.6-41.7\end{array}$ \\
\hline $\begin{array}{l}\text { Itching } \mathbf{X}^{2} \\
\text { (p-value) } \\
\text { OR-CI }\end{array}$ & $\begin{array}{c}0.12(p>0.05) \\
0.8: 0.2-2.6\end{array}$ & $\begin{array}{c}\text { FET } \\
1.89(\mathrm{p}>0.05) \\
0.3\end{array}$ & $\begin{array}{c}\mathbf{6 . 2}(\mathbf{p}<0.01) * \\
6.0: 1.3-28.4\end{array}$ & $\begin{array}{c}\text { FET } \\
1.19(\mathrm{p}>0.05) \\
2.1: .5-8.2\end{array}$ & $\begin{array}{c}\text { FET } \\
1.1(\mathrm{p}>0.05) \\
2.9: 0.4-23.9\end{array}$ & $\begin{array}{c}\text { FET } \\
0.22(\mathrm{p}>0.05) \\
1.4: 0.4-4.8\end{array}$ \\
\hline $\begin{array}{l}\text { Varicosities } \\
\mathrm{X}^{2}(\mathbf{p}-\mathrm{value}) \\
\text { OR-CI }\end{array}$ & $\begin{array}{c}\text { 7.1( }(<\mathbf{0 . 0 1})^{*} \\
3.3: 1.3-8.0\end{array}$ & $\begin{array}{c}0.004(\mathrm{p}>0.05) \\
1.0: 0.4-2.6\end{array}$ & $\begin{array}{c}0.5(\mathrm{p}>0.05) \\
0.75\end{array}$ & $\begin{array}{c}0.09(\mathrm{p}>0.05) \\
1.1: 0.5-2.6\end{array}$ & $\begin{array}{l}\mathbf{1 9 . 7}(<\mathbf{0 . 0 0 1})^{*} \\
0.14: 0.05-0.35\end{array}$ & $\begin{array}{l}1.9(>0.05) \\
0.6: 0.3-1.3\end{array}$ \\
\hline $\begin{array}{l}\text { Pig. spots } X^{2} \\
\text { (p-value) } \\
\text { OR-CI }\end{array}$ & $\begin{array}{c}0.09(>0.05) \\
0.9: 0.4-2.1\end{array}$ & $\begin{array}{c}0.006(\mathrm{p}>0.05) \\
0.96\end{array}$ & $\begin{array}{c}0.02(>0.05) \\
0.9: 0.4-2.3\end{array}$ & $\begin{array}{c}5.4(<0.05) \\
0.4: 0.14-0.87\end{array}$ & $\begin{array}{c}\mathbf{8 . 0}(<0.01)^{*} \\
0.3: 0.1-0.7\end{array}$ & $\begin{array}{c}10.0(<\mathbf{0 . 0 1}) * \\
6.4: 1.8-22.7\end{array}$ \\
\hline
\end{tabular}

Pig. Spots: Pigments spots

* Statistically significant

FET: Fisher Exact Test

OR : Odds Ratio

CI: Confidence Interval

PPE: Personal protective equipments 
Table 2 showed that physical examination of both groups revealed a significant higher percentage of skin disorders among street sweepers in comparison to administrative staff. Hair loss (19\% vs. 6.8\%), Scabies (15.1\% vs. 4\%), Frunculosis $(18.3 \%$ vs. $4.1 \%)$, Contact dermatitis ( $17.5 \%$ vs. $2.7 \%)$, Infected wound was (11\% vs. $1.4 \%)$, Varicosities $(25.4 \%$ vs. $6.8 \%)(\mathrm{p}<0.01)$ and finally, pigmentation spots $(19.8 \%$ vs. $8.1 \%)(\mathrm{p}<0.5)$.No significant differences were noticed regarding vitiligo, foot fissures and itching/ skin allergy.

Association between occurrence of some dermatological adverse effects and socio-demographic \& occupational factors of street sweepers were assessed. We found significant difference between those over 40 years vs. less than 40 considering hair loss , being over 40 years was associated with occurrence of varicosities $(\mathrm{X} 2=7.1$, OR: 3.3). Significant difference was detected between urban and rural residents regarding scabies, frunculosis , contact dermatitis , infected wound, foot fissures \& itching $(\mathrm{X} 2=14.5,12.6,11.3,12,8.8,6.2$ respectively) .Living in rural community was accompanied with increased risk of scabies (OR: 11.7), frunculosis (OR: 6.6), contact dermatitis (OR: 6.1), infected wound (OR: 16.7), foot fissures (OR: 8.3) and itching (OR: 6 ). Similarly, a significant difference was found between street sweepers according their educational level, illiteracy was strongly associated with scabies (X2=6.9, OR: 6.9), frunculosis (X2=11.3, OR: 9.2), contact dermatitis $(X 2=10.4, \mathrm{OR}: 8.6) \&$ infected wound $(X 2=9.6$, OR: 10). Significant differences were detected between those working for ten years or more and those who were not regarding varicosities and pigmentation spots ( $\mathrm{X} 2=19.7 \& 8$ ). Finally, there were strong association between not wearing PPE and contact dermatitis $(\mathrm{X} 2=7.6$, OR: 5.2), vitiligo ( $\mathrm{X} 2=6.2$, OR: 9.2), infected wound ( $\mathrm{X} 2=4.7$, OR: 4.8), foot fissures $(\mathrm{FET}=5.6, \mathrm{OR}: 5)$ and pigmentation spots (X2=10, OR:6.4) . 
Table (3): Comparison between street sweepers and control group regarding pulmonary function tests (PFTs).

\begin{tabular}{|c|c|c|c|c|}
\hline $\begin{array}{l}\text { Pulmonary } \\
\text { function tests }\end{array}$ & $\begin{array}{c}\text { Street } \\
\text { sweepers } \\
(\text { No=126) } \\
\end{array}$ & $\begin{array}{c}\text { Control } \\
\operatorname{group}(\mathrm{No}=74)\end{array}$ & $T$ - test & p-value \\
\hline $\begin{array}{l}\text { FEV1 } \\
\text { Range } \\
\text { Mean } \pm \text { SD }\end{array}$ & $\begin{array}{c}1.8-4.5 \\
3.3 \pm 0.68 \\
\end{array}$ & $\begin{array}{c}2-4.3 \\
3.8 \pm 0.65\end{array}$ & 5.38 & $(0.00) * *$ \\
\hline $\begin{array}{l}\text { FVC } \\
\text { Range } \\
\text { Mean } \pm \text { SD }\end{array}$ & $\begin{array}{c}2.8-5 \\
4.3 \pm 0.5\end{array}$ & $\begin{array}{c}3.6-4.8 \\
4.5 \pm 0.31\end{array}$ & 4.23 & $(\mathbf{0 . 0 0 )} * *$ \\
\hline $\begin{array}{l}\text { FEV1/FVC } \\
\text { ratio } \\
\text { Range } \\
\text { Mean } \pm \mathrm{SD}\end{array}$ & $\begin{array}{c}50-96.4 \\
77.1 \pm 11.9\end{array}$ & $\begin{array}{l}55.5-94.5 \\
84.1 \pm 11.1\end{array}$ & 4.09 & $(0.00) * *$ \\
\hline
\end{tabular}

**: Highly statistically significant

Table (3) showed significant decline of (FEV1, FVC and FEV1/FVC ratio) among street sweepers in comparison to the control group. 
Table (4): Association between respiratory manifestations, socio-demographic and occupational factors of street sweepers.

\begin{tabular}{|c|c|c|c|c|}
\hline Variables & Cough & Phlegm & Wheeze & $\begin{array}{c}\text { Shortness of } \\
\text { breath }\end{array}$ \\
\hline $\begin{array}{l}\text { Age group } \\
(\geq \mathbf{4 0} /<\mathbf{4 0} \text { yrs }): \\
X^{2} \quad(p-\text {-value }) \\
\text { OR } \\
\text { CI }\end{array}$ & $\begin{array}{c}0.11(\mathrm{p}>0.05) \\
1.1 \\
0.5-2.5\end{array}$ & $\begin{array}{c}0.26(\mathrm{p}>0.05) \\
1.2 \\
0.5-2.6\end{array}$ & $\begin{array}{c}0.08(\mathrm{p}>0.05) \\
0.9 \\
0.4-2.1\end{array}$ & $\begin{array}{c}0.006(\mathrm{p}>0.05) \\
1.0 \\
0.5-2.2\end{array}$ \\
\hline $\begin{array}{l}\text { Marital status } \\
\text { (single/ married): } \\
\mathrm{X}^{2} \quad \text { (p-value) } \\
\text { OR } \\
\mathrm{CI}\end{array}$ & $\begin{array}{c}0.032(\mathrm{p}>0.05) \\
1.1 \\
0.4-2.7\end{array}$ & $\begin{array}{c}0.004(\mathrm{p}>0.05) \\
1.0 \\
0.4-2.5\end{array}$ & $\begin{array}{c}0.006(\mathrm{p}>0.05) \\
0.9 \\
0.5-2.0\end{array}$ & $\begin{array}{c}0.09(\mathrm{p}>0.05) \\
1.14 \\
0.48-2.7\end{array}$ \\
\hline $\begin{array}{l}\text { Residence } \\
\text { (rural/ urban): } \\
\mathrm{X}^{2}(\mathrm{p} \text {-value) } \\
\text { OR } \\
\mathrm{CI}\end{array}$ & $\begin{array}{c}\mathbf{6 . 1}(\mathbf{p}<\mathbf{0 . 0 1}) * \\
0.4 \\
0.16-0.82\end{array}$ & $\begin{array}{c}\mathbf{7 . 0}(\mathbf{p}<\mathbf{0 . 0 1})^{*} \\
0.3 \\
0.15-0.76\end{array}$ & $\begin{array}{c}\mathbf{8 . 9}(\mathbf{p}<\mathbf{0 . 0 1}) * \\
0.3 \\
0.13-0.67\end{array}$ & $\begin{array}{c}\mathbf{8 . 9}(\mathbf{p}<\mathbf{0 . 0 1})^{*} \\
0.3 \\
0.13-0.67\end{array}$ \\
\hline $\begin{array}{l}\text { Educational level } \\
\text { (not educated/ } \\
\text { educated): } \\
\mathrm{X}^{2} \text { (p-value) } \\
\text { OR } \\
\mathrm{CI}\end{array}$ & $\begin{array}{c}\mathbf{7 . 8}(\mathbf{p}<\mathbf{0 . 0 1}) * \\
3.6 \\
1.4-9.0\end{array}$ & $\begin{array}{c}\mathbf{8 . 6}(\mathbf{p}<\mathbf{0 . 0 1})^{*} \\
3.8 \\
1.5-9.5\end{array}$ & $\begin{array}{c}7.9(\mathbf{p}<0.01) * \\
3.4 \\
1.4-8.3\end{array}$ & $\begin{array}{c}7.9(\mathbf{p}<0.01) * \\
3.4 \\
1.4-8.3\end{array}$ \\
\hline $\begin{array}{l}\text { Duration of } \\
\text { work }(\geq 10 /<10 \\
\text { yrs }) \text { : } \\
\left.X^{2} \quad \text { (p-value }\right) \\
\text { OR } \\
\text { CI }\end{array}$ & $\begin{array}{c}\mathbf{6 . 1}(\mathbf{p}<\mathbf{0 . 0 1}) * \\
5.6 \\
1.2-25.1\end{array}$ & $\begin{array}{c}\mathbf{6 . 5}(\mathbf{p}<\mathbf{0 . 0 1}) * \\
5.8 \\
1.3-26.2\end{array}$ & $\begin{array}{c}\mathbf{4 . 9}(\mathrm{p}<\mathbf{0 . 0 5}) * \\
3.9 \\
1.1-13.9\end{array}$ & $\begin{array}{c}\mathbf{4 . 9}(\mathbf{p}<\mathbf{0 . 0 5}) * \\
3.9 \\
1.1-13.9\end{array}$ \\
\hline $\begin{array}{l}\text { Wearing PPE(not } \\
\text { wearing/wearing): } \\
\mathrm{X}^{2} \text { (p-value) } \\
\text { OR } \\
\text { CI }\end{array}$ & $\begin{array}{c}\mathbf{5 . 7}(\mathbf{p}<\mathbf{0 . 0 1}) * \\
2.9 \\
1.2-7.0\end{array}$ & $\begin{array}{c}\mathbf{6 . 4}(\mathbf{p}<\mathbf{0 . 0 1}) * \\
3.1 \\
1.3-7.4\end{array}$ & $\begin{array}{c}\mathbf{7 . 9}(\mathbf{p}<\mathbf{0 . 0 1}) * \\
3.4 \\
1.4-8.3\end{array}$ & $\begin{array}{c}\mathbf{7 . 9}(\mathbf{p}<\mathbf{0 . 0 1}) * \\
3.4 \\
1.4-8.3\end{array}$ \\
\hline
\end{tabular}

* Statistically significant

PPE: Personal protective equipments

OR : Odds Ratio

CI: Confidence Interval 
Table (4) showed no significant differences between street sweepers showing respiratory manifestations or not considering their age or marital status. On the contrary, we found strong association between urban residency and the occurrence of chronic cough (X2=6.1,OR: 0.4) ,phlegm(X2 =7,OR: $0.3)$,wheeze and shortness of breath (X2=8.9,OR : 0.3) (rural residency act as a protective factor against respiratory manifestation).

Illiteracy was associated with the occurrence of chronic cough $(\mathrm{X} 2=7.8$, OR: 3.6), expectoration of phlegm $(\mathrm{X} 2=8.6$, OR: 3.8$)$, wheeze and shortness of breath (X2=7.9,OR: 3.4). Working for 10 years or more is associated with development of chronic $\operatorname{cough}(\mathrm{X} 2=6.1, \mathrm{OR}: 5.6)$, expectoration of phlegm (X2=6.5, OR: 5.8), wheeze and shortness of breath $(\mathrm{X} 2=4.9$, OR: 3.9). Finally, the study revealed an association between not wearing personal protective clothes and the development of chronic cough (X2=5.7, OR: 2.9), expectoration of phlegm $(\mathrm{X} 2=6.4$, OR: 3.1) wheezes and shortness of breath (X2=7.9, OR: 3.4 ).

\section{Discussion}

This study is the first study on male street sweeper's workers in Qualubya governorate, as far as the author knows. The aim of this study was to recognize respiratory and skin disorders due to work practices among street sweepers.

Street sweeping and collecting waste is a job that is usually performed by poor people, mostly males, being responsible for families of large size, and with low educational and socioeconomic levels. This applies to Egypt (Abd El Wahab et al, 2014) and other developing countries (Nagaraj et al, 2004).

There was no statistically significant difference between sweeper's workers and control group regarding age, duration of work (Table 1). In Addition, there were no past medical or family histories of respiratory illnesses, chest operations or injuries among the participants.

Our findings detected that dermatological examination were higher among street sweepers( Table 2) than other previous Egyptian researches. In Alexandria, a cross-sectional study was conducted among Municipal Solid Waste Workers (MSWWs) and they estimated the prevalence of scabies, dermatitis, frunculosis, infected wound, foot cracks and vitiligo to be $(4.2 \%, 5.4 \%, 0.9 \%$, $1.7 \%, 1.2 \%$ and $0.9 \%$ respectively). About $3 \%$ of the MSWWs were com- 
plaining of pruritus due to skin allergy (Abd El Wahab et al., 2014).

On the contrary, the results of our study came in agreement with other study done in Beni-suef, Egypt by Ewis, et al. 2013 who studied the possible work-related morbidities among street sweepers and waste collectors. They found that skin irritations occurred in (13.8\%) of street sweepers, due to direct contact with wastes without protective devices. This was similar to our findings (17.2\% of study group had contact dermatitis). The difference between our findings and Ewis, et al. 2013 and that of Abd El Wahab et al.2014, could be attributed to the type of governorate and socio-demographic variations (Alexandria is an urban governorate while Qualubya and Beni-suef are semi-urban ones with poor hygiene level, poor working conditions, long working hours, lack of cleaning measures, high working intensity and poor environment).

Our study found strong association between working for ten years or more and the occurrence of varicosities and pigmentation spots. Rural residency and illiteracy were strongly associated with the occurrence of most of skin manifestations. Being over 40 years of age was associated with occurrence of varicosities (Table 2).

Occurrence of these symptoms in street sweepers can be explained by the role of UV damage which is the most common problems among sweepers. Excessive exposure to UV radiation can result in chronic harmful effects on the skin, eye and immune system (Young and Walker, 2005). Besides skin photo aging caused mainly by UV exposure, many other skin manifestations can be connected with their long periods of standing and heavy manual work which led to varicosity (Yan et al., 2015).

As regards PFTs of street sweepers, our study showed significant decline of (FEV1, FVC and FEV1/FVC ratio) in comparison to the control group (Table 3).This resembles to another study performed in Egypt by Abd El Wahab et al., 2014 .In Pakistan, a cross sectional study found that PFTs had lower values among the sweepers group when compared to the control one and the pattern of pulmonary airway obstruction was proportional to the duration of occupational exposure to dust (Anwar et al., 2013). Also, we are in agreement with several other studies like Athanasiou et al., 2010 and Johncy et al., 2013. 
Another Indian study found a significant decrease in FEV1and FEV1/ FVC ratio $<80 \%$ measured for street sweepers indicating obstructive pulmonary disease (Shadab et al., 2013). The same results was found in a study done in Turkish which showed that exposure to bio-aerosols can result in ventilatory disorders, and differences in spirometric findings between study and control groups (Issever et al., 2002). Similarly, Ranjan et al. in their study which was conducted on landfill site workers, showed a significant increase in lung functional impairments in Municipal Solid Waste Workers (MSWWs) (Ranjan et al, 2005). Moreover, Zuskin et al. reported significant decrements in FEV1 and FVC values as compared with a referent group (Zuskin et al., 1996).

A comparative study was conducted upon waste collectors in Western $\mathrm{Mu}$ nicipality in Mansoura, Egypt. Longer duration of employment was independently associated with impaired FEV1/ FVC, FEV1 and FVC (Abou El wafa et al., 2012). However a study in Calabar, Nigeria did not found a statistically significant decrease in FVC, FEV1, and PEFR among street cleaners when compared to control subjects (Nku et al. ,2005).
This decline in PFTs may be due to the exposure to street sweeping dust. Respiratory symptoms may occur as result of pollutants from the street, car traffics, and exhaust engine fuel which results to the production of fumes and gases in addition to organic and inorganic dust particles. The problem was aggravated by negligence of wearing PPEs as masks during work. They work outdoor for $>8$ hours daily, and this expose them to large amounts of dust, predisposing them to the development of such respiratory illnesses and COPD.

Several studies have shown high prevalence of cough among street sweepers. A study done in Cairo, Egypt showed that cough as a symptom related to chronic bronchitis was significantly more common among street sweepers $(17.5 \%)$ than among office workers (5.8\%) (Mostafa et al., 2015). Another study done in Beni-Suef, Egypt revealed that cough was significantly more frequent among those working in street sweeping and waste collection than clerks $(18.1 \%$ vs. $7.1 \%$, respectively) (Ewis et al., 2013).

Similarly, higher prevalence of respiratory disorders among street sweepers than among the control group, mainly cough, catarrh, and sneezing was found in Nigeria by Nku et al., 2005. 
Two studies in India detected a significant higher prevalence of chronic respiratory diseases among sweepers than among the comparison group (Yogesh and Sanjay, 2008 a; Yogesh and Sanjay ,2008b).

Also the same results were established in a cross-sectional study on street sweepers vs. street vegetable sellers in Ilala Municipality. Respiratory symptoms in that study among the exposed group compared to the control were: cough $(54.9 \%$ vs. $12.9 \%)$ phlegm (39.2 vs. $7.1 \%)$, wheezes $(32.4 \%$ vs. $14.1 \%)$.Street sweeping dust was the main factor leading to cough, phlegm and wheezes (Stambuli,2012) .While in another study done in Ethiopia, the overall prevalence of respiratory symptoms were $40.7 \%$.The prevalence of cough was $35.7 \%$, wheezes $21.2 \%$, phlegm $44 \%$, chest illness $7.3 \%$ and breathlessness $29.2 \%$ among solid waste collectors in Yeka sub city (Emiru1 et al.,2017).

Association between respiratory manifestations and socio demographic and occupational factors among street sweepers were assessed and we found a strong association between working for 10 years or more, urban residency, educational level and not wearing PPEs on one side and development of chronic cough, expectoration of phlegm, wheezes and shortness of breath on the other side (Table 4). This might be due to the exposure to high dust level without proper preventive mechanisms. The same results were detected in other studies (Nku et al., 2005; Aguwa et al., 2007; Tamara et al., 2008).

\section{Recommendations}

There is an urgent stressful need to take care of sweeping workers as a vulnerable group for many health hazards. Pre- employment, periodic medical examination and individual health cards should be mandatory for them. All of them should be under the umbrella of governmental health insurance. Compulsory utilization of PPEs with close supervision for all workers should be carried on with continuous training for proper use of protective equipments. Also there is an important role for both engineering and legislative measures in minimizing these occupational hazards.

\section{Conflict of interest}

None.

\section{References}

1. Abd El-Wahab E, Eassa S, Lotfi S, El Masry S, Shatat H, et al. (2014): Adverse Health Problems among Municipality Workers in Alexandria (Egypt). Int J Prev Med; 5(5): 545-56. 
2. Abou-El Wafa HS, El-Bestar SF, El-Gilany AH and Awad El-Toraby (2012):Musculoskeletal disorders among municipal solid waste collectors in Mansoura, Egypt: a cross-sectional study. BMJ; 2(5): e001338.

3. Abou-El Wafa HS, El-Bestar SF, El-Gilany AH, Awad El-Toraby et al. (2014): Respiratory Disorders among Municipal Solid Waste Collectors in Mansoura, Egypt: A Comparative Study. Arch Environ Occup Hlth;69(2):100-6

4. Aguwa EN, Okeke TA and Asuzu MC (2007): The Prevalence of Occupational Asthma and Rhinitis among Woodworkers in South-West Nigeria. Tanzan J Health Res; 9 (1): 52 - 5.

5. Anwar SK, Mehmood N, Nasim N, Khurshid M and Khurshid B (2013): Sweeper's lung disease: a cross sectional study of an overlooked illness among sweepers of Pakistan. Intern J of COPD; 8:1937.

6. Athanasiou M, Makrynos Gand Dounias G (2010): Respiratory health of municipal solid waste workers. Occup Med (Lond); 60:61823.

7. Chandler TD and Feuille P (1994): Cities, unions, and the privatization of sanitation services. J Labor Res; 15(1): 53-71.

8. Cointreau-Levine S (1995): Occupational and environmental health issues of solid waste management [cited on 2004 Jan 5]. Available at http://wgbis.ces.iisc.ernet.in/energy/paper/ privatesector/collection.html.

9. CointreauLevine S, Listorti $\mathrm{J}$ and Furedy $\mathrm{C}$ (1998): Solid waste. In: Herzstein JA, Bunn WB, Fleming LE, Harrington JM, Jeyaratnam J, Gardner IR,editors. International Occupational and Environmental Medicine. 1st ed.St. Louis: Mosby .p: 62032.

10. CointreauLevine S (2006): Occupational and Environmental Health Issues of Solid Waste Management. Special Emphasis on Middle and Lower Income Countries. Urban papers. Washington D.C: World Bank Group, Urban Sector Board; Available at: http://www worldbank.org/INTUSWM/Resources/up2.pdf. (Last cited 2012 Aug 01).
11. Comstock GW, Tockman MS, Helsing KJ and Hennesy KM (1979): Standardized respiratory questionnaires: Comparison of the old with the new. Am Rev Respir Dis; 119(1):45-53.

12. Emiru1 Z, Gezu M, Chichiabellu T, Dessalegn L and Anjulo A (2017): Assessment of respiratory symptoms and associated factors among solid waste collectors in Yeka Sub City, Addis Ababa, Ethiopia.Journal of Public Health and Epidemiology; 9(6): 189-97

13. Ewis AA, Rahma MA, Mohamed ES, Hifnawy TM and Arafa AE (2013): Occupational HealthRelated Morbidities among Street Sweepers and Waste Collectors at Beni-Suef, Egypt. EJOM; 37 (1): 79-94

14. GBD (Global Burden of Disease) (2010): Report from Disability Adjusted Life Years (DALYs) at Health Data website (Cited 2014 June 26); Available at: http://vizhub.healthdata.org/ gbdcompare.

15. Issever H, Hulya G and Erelel M (2002): Health problem of garbage collectors in Istanbul. Indoor Built Environ J; 11: 293- 301.

16. Johncy SS, Dhanyakumar G, Samuel TV, Ajay KT and Bondade SY (2013): Acute Lung Function Response to Dust in Street Sweepers. Journal of Clinical and Diagnostic Research (JCDR); 7(10):2126-9.

17. Krajewski JA, Tarkowski S, Cyprowski M, Szarapinska-Kwaszewska J and Dudkiewicz B (2002):Occupational exposure to organic dust associated with municipal waste collection and management. Int J Occup Med Environ Hlth; 15:289-301.

18. Mostafa N, Abdel-Hamid M and Al Bagoury L (2015): Work-related respiratory disorders among street sweepers in Cairo, Egypt, a comparative study. The Egyptian Journal of Community Medicine;37 (1): 79-94.

19. Nagaraj C, Shivram C and Jayanthkumar NN (2004): A study of morbidity and mortality profile of sweepers working under Banglore City Corporation. Indian J Occup Environ Med; 08:11-6. 
20. Nayak S, Shenoi S, Kaur G, Bisen N, Purkayastha A, et al. (2013): Dermatologic evaluation of street sanitation workers. Indian J Dermatol; 58(3): 246.

21. Nku CO, Peters EJ, Estiet Al, Oku O and Osim EE (2005): Lung function, Oxygen Saturation and Symptoms among Street Sweepers in Calabar Nigeria. Niger J physiol Sci; 20 (1-2): 79-84.

22. Ranjan Ray M, Roychouhury S, Gopeshwar $M$ and Senjuti Roy TL(2005):Respiratory and general health impairments of workers employed in a municipal solid waste disposal at an open landfill site in Delhi. Intern J Hyg Envi Hlth; 208: 255-62.

23. Seaton A (1995): Silicosis In: Morgan WKC, Seaton A, eds. Occupational lung diseases. 3rd ed. Philadelphia: WB Saunders; 222-37.

24. Shadab M, Agrawal DK, Ahmad Z and Aslam M (2013):A cross sectional study of Pulmonary Function Tests in street cleaners in Aligarh, India. Biomedical Research; 24 (4): 449-52.

25. Tamara S, Dorothee S, Verena R, Beate P, Ulrich R et al. (2008): Contribution of Smoking and Air Pollution Exposure in Urban areas to Social Differences in Respiratory health. BMC Public Health; 8: 179.

26. Yan Y, Wang X, Jianbo Wu J and Xu L (2015): Occupational skin diseases and prevention among sanitation workers in China. Afr Hlth Sci ; 15(3): 768-75.

27. Young AR and Walker SL (2005): UV radiation, vitamin $\mathrm{D}$ and human health: an unfolding controversy introduction. Photochem Photobiol; 81(6):1243-5.

28. Yogesh DS and Sanjay PZ (2008)a : A Study of Morbidity Pattern in Street Sweepers: A Crosssectional Study. Indian J Community Med; 33(4): 224-8.

29. Yogesh DS and Sanjay PZ (2008)b: Respiratory morbidity among street sweepers working at Hanumannagar Zone of Nagpur Municipal Corporation, Maharashtra. Indian J Public Health; 52(3): 147-9

30. Zuskin E, Mustajbegovic J, Schachter E, Neil J , Kern D et al. (1996): Airway function and respiratory symptoms in sanitation workers. $\mathrm{J}$ Occup Env Med; 38(5):522-7. 
